International Journal of Current Microbiology and Applied Sciences ISSN: 2319-7706 Volume 9 Number 2 (2020)

\title{
Bio-efficacy Trials of Spinotetram 0.8\% GR against Yellow Stem Borer, Scirpophaga incertulas and Leaf Folder, Cnaphalocrocis medinalis Infesting Rice
}

\author{
Snigdha Samanta, Mritunjoy Barman, R. Nihal* and Arunava Samanta \\ Department of Agricultural Entomology, Bidhan Chandra Krishi Viswavidyalaya, \\ Mohanpur, Nadia-741252, West Bengal, India \\ *Corresponding author
}

\section{A B S T R A C T}

The present investigation was conducted in summer 2018 at BCKV

\section{Keywords}

Rice, Stem borer, Leaf folder, Bio-

efficacy,

Spinotetram $0.8 \%$

GR.

Article Info

\section{Accepted:}

20 January 2020

Available Online:

10 February 2020

Teaching Farm, Mandouri, Nadia, West Bengal, to evaluate the efficacy insecticide, viz., Spinotetram $0.8 \%$ GR at different doze rates against yellow stem borer, Scirpophaga incertulas and Rice leaf folder, Cnaphalocrosis medinalis infesting rice. The pooled data on efficacy of different treatment schedules of Spinotetram $0.8 \%$ GR against major insect pests of Rice i.e. Stem borer and Leaf folder. All the treated plots provided significant reduction of pest infestation along with significant yield increase but the best protection was obtained from the plots treated with Spinotetram 0.8\% GR @ 70g/ha and Spinotetram 0.8\% GR @ 65g/ha followed by Spinotetram 0.8\% GR @ 60g/ha \& Cartap Hydrochloride 4\% GR @ $1000 \mathrm{~g} / \mathrm{ha}$. All the treatments were on par with each other and significantly superior over the other comparative treatments and untreated control regarding pest control.

\section{Introduction}

Rice is the most important food crop in India and on research, and production priority for national food security. Rice contains protein name gluten $(8.1 \%)$, Vitamins, minerals, fibers (2.2\%) and lots of carbohydrates (77.1\%) with a total of 349 calories. Rice crop is highly sensitive for several insect pests Singh and Singh, (2015), Singh and Singh,
(2017). The major factors that have contributed towards changes in the pest scenario are extensive cultivation of high yielding varieties, growing of varieties lacking resistance to major pests, intensified rice cultivation throughout the year providing constant niches for pest multiplication, imbalanced use of fertilizers, particularly application of high levels of nitrogen, nonjudicious use of insecticides resulting in pest 
resistance to insecticides, and resurgence of pests and out breaks of minor pests Prakash et. al., (2014) . The number of insects pests recorded during this survey clearly shows the damage intensity caused by insects, this survey report will support for making the decision for management of major insects.

Insects are the most diverse group of animals living on earth. They are undoubtedly the most adaptable form of life as their number exceeds that of any other category. Among the major insect pests of rice are stem borers like yellow stem borer-YSB (Scirpophaga incertulas), belong to order Lepidoptera is the most destructive pest found all over the world. The presence of these insects in our field is easily identified by "dead heart" or "white ear" in hills at vegetative stage and panicle at reproductive stages respectively Sulagitti et.al., (2018). The Cnaphalocrocis medinalis (Leaf folder) belongs to order Lepidoptera also observed during both stages and feed inside the leaves make a fold. Gundhi bug (Leptocorisa oratorius) is a serious pest of rice and it reduces yield up to $30 \%$. Both adult and nymphs feed on grains at the milking stage. The population of bugs increases at the end of the rainy seasons Singh and Singh, (2017).

Among the various strategies adopted to combat the pest of rice, insecticides are the first line of defence. Most of the insecticides used on agricultural crops are based on quit limited number of chemically different classes out of them the most important inorganic insecticides that are used against the pest on rice belongs to organophosphate and synthetic pyrethroids. Therefore an effort has been made in present investigation to evaluate the new molecules of chemical insecticides such as Spinotetram $0.8 \%$ GR at different doze rates supplied by Dow Agrosciences India Pvt. Ltd. against rice yellow stem borer and leaf folder

\section{Materials and Methods}

A field experiment was conducted at BCKV Teaching Farm, Mandouri, Nadia, West Bengal to evaluate insecticide molecules against yellow stem borer and Leaf folder in rice variety "GB-3"comprising six treatments and three replication in randomized block design during summer 2018. Two rounds of applications were given one at active tillering upon the appearance of leaf folder when the pest status reached beyond ETL (one damaged leaf per hill) and second at panicle initiation stage.

The observations on incidence of yellow stem borer were taken with respect to Dead Hearts (DH) \& White ear head (WEH) count. Whereas, leaf folder incidence was taken on the basis of number of larvae. Observations to be taken on pest's population in each plot before application and 3, 7, 10, 14 \& 21 days after each application. In each plot 10 hills were randomly selected and the percentage of dead hearts \& white ears per hill were observed randomly from top to bottom for calculating the insect pest population. The obtained mean values are used for ANOVA. The populations of natural enemies were recorded before and after the imposition of first and second application.

\section{Results and Discussion}

\section{Bio-efficacy of Spinotetram $0.8 \%$ GR against Leaf folder of Rice in summer $\mathbf{2 0 1 8}$}

Before the imposition of the treatments the number of no. of larvae ranged from 0.84 to 1.08 larvae per hill. After the imposition of treatments the number of larvae decreased gradually till 21 days and all the treatments were significantly superior to the untreated control. At 21 days after the application no infestation i.e. 0.00 and 0.00 was recorded in the treatments Spinotetram 0.8\% GR @ 
70g/ha and Spinotetram 0.8\% GR @ 65g/ha (100\% and $100 \%$ reduction over control). These were followed by Spinotetram $0.8 \%$ GR @ 60g/ha, Cartap Hydrochloride 4\% GR @ 1000g/ha and Chlorantraniliprole $0.4 \%$ GR @ 40g/ha which recorded $0.05,0.10$ and 0.14 larvae/hill $(95.83,91.66 \& 88.34 \%$ reduction over control). In untreated control the maximum number of larvae was 1.20 per hill was recorded (Table 1).

Similar trend was observed after the second application. Before the imposition of treatments the number of larvae i.e. the leaf folder infestation ranged from 0.98 to 1.20 larvae per hill. After the imposition of treatments the population of leaf folder decreased gradually till 21 days and all the treatments were significantly superior to the untreated control.

At 21 days after the application the least leaf folder infestation i.e. 0.00 and 0.00 larvae per hill was recorded in the treatments Spinotetram 0.8\% GR @ 70g/ha and Spinotetram 0.8\% GR @ 65g/ha (100\% and $100 \%$ reduction over control respectively). These were followed by Spinotetram $0.8 \%$ GR @ 60g/ha, Chlorantraniliprole 0.4\% GR @ 40g/ha and Cartap Hydrochloride 4\% GR @ 1000g/ha recorded $0.05,0.15$ and 0.18 larvae/hill (96.06, 88.18 and $85.82 \%$ reduction over control). In untreated control the maximum number of larval population of 1.27 per hill was recorded (Table 2).

\section{Bio-efficacy of Spinotetram $0.8 \%$ GR} against Stem Borer of Rice in summer 2018

Before the imposition of the treatments the stem borer infestation the percentage of dead hearts ranged from $12.58 \%$ to $13.56 \%$. After the imposition of treatment the percentage of dead hearts decreased gradually till 21 days and all the treatments were significantly superior than the untreated control. At 21 days after the application the least percentage of dead hearts $0.00 \%$ and $0.00 \%$ was recorded in the Spinotetram 0.8\% GR @ 70g/ha and Spinotetram 0.8\% GR @ 65g/ha. (100\% and $100 \%$ reduction over control) and were on par with Spinotetram 0.8\% GR @ 60g/ha which recorded $0.05 \%$ damaged leaves/hill $(99.66 \%$ reduction over control). These were followed by $3.16 \%$ and $3.24 \%$ in Chlorantraniliprole 0.4\% GR @ 40g/ha and Cartap Hydrochloride 4\% GR @ 1000g/ha. (Table 3).

Similar trend was observed after the second application imposition. Before the imposition of treatments the stem borer population i.e. the percentage of white earheads ranged from $9.25 \%$ to $13.05 \%$ per plant. At 21 days after the second application the least percentage of white earheads ranged $0.00 \%, 0.00 \%$ and $0.00 \%$ was recorded in the treatments Spinotetram 0.8\% GR @ 70g/ha, Spinotetram 0.8\% GR @65g/ha \& Spinotetram 0.8\% GR @ 60g/ha (100\%,100\% and 100\% reduction over control). These were followed by $1.15 \%$ and $1.36 \%$ in Cartap Hydrochloride $4 \%$ GR @ 1000g/ha and Chlorantraniliprole 0.4\% GR @ 40g/ha. In untreated control the maximum percentage of white ear heads ranged $13.60 \%$ was recorded. (Table 4).

\section{Effect of different insecticidal treatments on the yield}

All the treatments were superior over the untreated check. The yield data of Rice grain was highest 35.24 and $34.89 \mathrm{q} / \mathrm{ha}$ in Spinotetram $\quad 0.8 \% \quad$ GR @ 70g/ha and Spinotetram 0.8\% GR @ 65g/ha. Both the treatments were on par Spinotetram 0.8\% GR @ 60g/ha and were significantly superior to the standard checks. These were followed by $25.18 \mathrm{q} / \mathrm{ha}$ in Cartap Hydrochloride 4\% GR @ $1000 \mathrm{~g} / \mathrm{ha}$ and $25.14 \mathrm{q} / \mathrm{ha}$ in Chlorantraniliprole 0.4\% GR @40g/ha. In untreated check the lowest yield 23.85 q/ha was recorded (Table 5). 
Table.1 Bioefficacy of Spinotetram $0.8 \%$ GR on Leaf folder (First application)

\begin{tabular}{|c|c|c|c|c|c|c|c|c|c|c|}
\hline \multirow{2}{*}{$\begin{array}{l}\text { Sr. } \\
\text { No. }\end{array}$} & \multirow[t]{2}{*}{ Treatment } & \multirow{2}{*}{$\begin{array}{l}\text { Dose g } \\
\text { a.i/ha }\end{array}$} & \multirow{2}{*}{$\begin{array}{l}\text { Formulation } \\
\text { (kg/ac) }\end{array}$} & \multicolumn{6}{|c|}{ Mean No. of leaf folder larvae / hill. } & \multirow{2}{*}{$\begin{array}{l}\text { \% reduction at } 15 \\
\text { days after } \\
\text { application }\end{array}$} \\
\hline & & & & $\mathbf{P T}^{\wedge}$ & 3 & 7 & 10 & 14 & 21 & \\
\hline T1 & Spinotetram $0.8 \%$ GR & 60 & 3 & $\begin{array}{l}1.05 \\
(1.24)\end{array}$ & $\begin{array}{l}0.69 \\
(1.09)\end{array}$ & $\begin{array}{l}0.49 \\
(0.99)\end{array}$ & $\begin{array}{l}0.38 \\
(0.94)\end{array}$ & $\begin{array}{l}0.18 \\
(0.82)\end{array}$ & $\begin{array}{l}0.05 \\
(0.74)\end{array}$ & 95.83 \\
\hline $\mathbf{T 2}$ & Spinotetram $0.8 \%$ GR & 65 & 3.25 & $\begin{array}{l}1.08 \\
(1.25)\end{array}$ & $\begin{array}{l}0.63 \\
(1.06)\end{array}$ & $\begin{array}{l}0.45 \\
(0.97)\end{array}$ & $\begin{array}{l}0.32 \\
(0.90)\end{array}$ & $\begin{array}{l}0.10 \\
(0.77)\end{array}$ & $\begin{array}{l}0.00 \\
(0.71)\end{array}$ & 100 \\
\hline T3 & Spinotetram $0.8 \%$ GR & 70 & 3.5 & $\begin{array}{l}0.94 \\
(1.20)\end{array}$ & $\begin{array}{l}0.58 \\
(1.04)\end{array}$ & $\begin{array}{l}0.36 \\
(0.93)\end{array}$ & $\begin{array}{l}0.28 \\
(0.88)\end{array}$ & $\begin{array}{l}0.07 \\
(0.75)\end{array}$ & $\begin{array}{l}0.00 \\
(0.71)\end{array}$ & 100 \\
\hline T4 & Chlorantraniliprole $0.4 \%$ GR & 40 & 4 & $\begin{array}{l}1.00 \\
(1.22)\end{array}$ & $\begin{array}{l}0.92 \\
(1.19)\end{array}$ & $\begin{array}{l}0.71 \\
(1.10)\end{array}$ & $\begin{array}{l}0.57 \\
(1.03)\end{array}$ & $\begin{array}{l}0.38 \\
(0.94)\end{array}$ & $\begin{array}{l}0.14 \\
(0.80)\end{array}$ & 88.34 \\
\hline T5 & Cartap Hydrochloride 4\% GR & 1000 & 10 & $\begin{array}{l}0.84 \\
(1.16)\end{array}$ & $\begin{array}{l}0.79 \\
(1.14)\end{array}$ & $\begin{array}{l}0.68 \\
(1.09)\end{array}$ & $\begin{array}{l}0.50 \\
(1.00)\end{array}$ & $\begin{array}{l}0.30 \\
(0.89)\end{array}$ & $\begin{array}{l}0.10 \\
(0.77)\end{array}$ & 91.66 \\
\hline T6 & Untreated control & & & $\begin{array}{l}0.98 \\
(1.22)\end{array}$ & $\begin{array}{l}1.10 \\
(1.26)\end{array}$ & $\begin{array}{l}1.15 \\
(1.28)\end{array}$ & $\begin{array}{l}1.18 \\
(1.30)\end{array}$ & $\begin{array}{l}1.20 \\
(1.30)\end{array}$ & $\begin{array}{l}1.20 \\
(1.30)\end{array}$ & - \\
\hline & Sem & & - & 0.04 & 0.03 & 0.03 & 0.02 & 0.02 & 0.01 & - \\
\hline & CD $(p=0.05)$ & & - & NS & 0.11 & 0.09 & 0.07 & 0.05 & 0.03 & - \\
\hline & CV \% & & - & NS & 5.34 & 4.71 & 4.18 & 3.20 & 2.00 & - \\
\hline
\end{tabular}

*Mean of three replications

$\mathrm{PT}^{\wedge}$ Pretreatment.

Values in parenthesis are ArcSine transformed. 
Table.2 Bioefficacy of Spinotetram $0.8 \%$ GR on Leaf folder (Second application)

\begin{tabular}{|c|c|c|c|c|c|c|c|c|c|c|}
\hline \multirow{2}{*}{$\begin{array}{l}\text { Sr. } \\
\text { No. }\end{array}$} & \multirow[t]{2}{*}{ Treatment } & \multirow{2}{*}{$\begin{array}{l}\text { Dose g } \\
\text { a.i/ha }\end{array}$} & \multirow{2}{*}{$\begin{array}{l}\text { Formulation } \\
\text { (kg or } \mathrm{ml} / \mathrm{ha} \text { ) }\end{array}$} & \multicolumn{6}{|c|}{ Mean No. of leaf folder larvae / hill. } & \multirow{2}{*}{$\begin{array}{l}\text { \% reduction at } 15 \\
\text { days after } \\
\text { application }\end{array}$} \\
\hline & & & & $\mathbf{P T}^{\wedge}$ & 3 & 7 & 10 & 14 & 21 & \\
\hline T1 & Spinotetram $0.8 \%$ GR & 60 & 3 & $\begin{array}{l}0.97 \\
(1.21)\end{array}$ & $\begin{array}{l}0.74 \\
(1.11)\end{array}$ & $\begin{array}{l}0.58 \\
(1.04)\end{array}$ & $\begin{array}{l}0.36 \\
(0.93)\end{array}$ & $\begin{array}{l}0.15 \\
(0.81)\end{array}$ & $\begin{array}{l}0.05 \\
(0.74)\end{array}$ & 96.06 \\
\hline $\mathbf{T 2}$ & Spinotetram $0.8 \%$ GR & 65 & 3.25 & $\begin{array}{l}1.08 \\
(1.25)\end{array}$ & $\begin{array}{l}0.65 \\
(1.07)\end{array}$ & $\begin{array}{l}0.45 \\
(0.97)\end{array}$ & $\begin{array}{l}0.25 \\
(0.87)\end{array}$ & $\begin{array}{l}0.06 \\
(0.75)\end{array}$ & $\begin{array}{l}0.00 \\
(0.71)\end{array}$ & 100 \\
\hline T3 & Spinotetram $0.8 \%$ GR & 70 & 3.5 & $\begin{array}{l}1.10 \\
(1.26)\end{array}$ & $\begin{array}{l}0.62 \\
(1.06)\end{array}$ & $\begin{array}{l}0.43 \\
(0.96)\end{array}$ & $\begin{array}{l}0.20 \\
(0.84)\end{array}$ & $\begin{array}{l}0.03 \\
(0.73)\end{array}$ & $\begin{array}{l}0.00 \\
(0.71)\end{array}$ & 100 \\
\hline T4 & Chlorantraniliprole $0.4 \%$ GR & 40 & 4 & $\begin{array}{l}1.14 \\
(1.28)\end{array}$ & $\begin{array}{l}0.85 \\
(1.16)\end{array}$ & $\begin{array}{l}0.73 \\
(1.11)\end{array}$ & $\begin{array}{l}0.58 \\
(1.04)\end{array}$ & $\begin{array}{l}0.28 \\
(0.88)\end{array}$ & $\begin{array}{l}0.15 \\
(0.81)\end{array}$ & 88.18 \\
\hline T5 & Cartap Hydrochloride 4\% GR & 1000 & 10 & $\begin{array}{l}0.98 \\
(1.22)\end{array}$ & $\begin{array}{l}0.89 \\
(1.18)\end{array}$ & $\begin{array}{l}0.69 \\
(1.09)\end{array}$ & $\begin{array}{l}0.51 \\
(1.00)\end{array}$ & $\begin{array}{l}0.24 \\
(0.86)\end{array}$ & $\begin{array}{l}0.18 \\
(0.82)\end{array}$ & 85.82 \\
\hline \multirow[t]{4}{*}{ T6 } & Untreated control & & & $\begin{array}{l}1.20 \\
(1.30)\end{array}$ & $\begin{array}{l}1.23 \\
(1.32)\end{array}$ & $\begin{array}{l}1.25 \\
(1.32)\end{array}$ & $\begin{array}{l}1.25 \\
(1.32)\end{array}$ & $\begin{array}{l}1.27 \\
(1.33)\end{array}$ & $\begin{array}{l}1.27 \\
(1.33)\end{array}$ & - \\
\hline & Sem & & - & 0.04 & 0.03 & 0.03 & 0.02 & 0.01 & 0.01 & - \\
\hline & CD $(p=0.05)$ & & - & - & 0.11 & 0.09 & 0.07 & 0.04 & 0.03 & - \\
\hline & CV \% & & - & - & 5.20 & 4.78 & 4.13 & 2.77 & 2.08 & - \\
\hline
\end{tabular}


Table.3 Bioefficacy of Spinotetram $0.8 \%$ GR on Stem borer (First application)

\begin{tabular}{|c|c|c|c|c|c|c|c|c|c|c|}
\hline \multirow{2}{*}{$\begin{array}{l}\text { Sr. } \\
\text { No. }\end{array}$} & \multirow[t]{2}{*}{ Treatment } & \multirow{2}{*}{$\begin{array}{l}\text { Dose g } \\
\text { a.i/ha }\end{array}$} & \multirow{2}{*}{$\begin{array}{l}\text { Formulation } \\
\text { (kg or } \\
\mathrm{ml} / \mathbf{h a})\end{array}$} & \multicolumn{6}{|c|}{ Percentage of Dead heart (\%) } & \multirow{2}{*}{$\begin{array}{l}\text { \% reduction at } 15 \\
\text { days after } \\
\text { application }\end{array}$} \\
\hline & & & & $\mathbf{P T}^{\wedge}$ & 3 & 7 & 10 & 14 & 21 & \\
\hline $\mathbf{T 1}$ & Spinotetram $0.8 \%$ GR & 60 & 3 & $\begin{array}{l}12.58 \\
(21.12)\end{array}$ & $\begin{array}{l}9.85 \\
(18.70)\end{array}$ & $\begin{array}{l}6.52 \\
(15.31)\end{array}$ & $\begin{array}{l}5.57 \\
(14.21)\end{array}$ & $\begin{array}{l}2.51 \\
(9.96)\end{array}$ & $\begin{array}{l}0.05 \\
(4.25)\end{array}$ & 99.66 \\
\hline $\mathbf{T 2}$ & Spinotetram $0.8 \%$ GR & 65 & 3.25 & $\begin{array}{l}13.28 \\
(21.69)\end{array}$ & $\begin{array}{l}9.24 \\
(18.10)\end{array}$ & $\begin{array}{l}5.98 \\
(14.68)\end{array}$ & $\begin{array}{l}5.40 \\
(13.99)\end{array}$ & $\begin{array}{l}1.85 \\
(8.79)\end{array}$ & $\begin{array}{l}0.00 \\
(4.05)\end{array}$ & 100.00 \\
\hline T3 & Spinotetram $0.8 \%$ GR & 70 & 3.5 & $\begin{array}{l}12.82 \\
(21.40)\end{array}$ & $\begin{array}{l}8.56 \\
(17.52)\end{array}$ & $\begin{array}{l}6.06 \\
(14.84)\end{array}$ & $\begin{array}{l}5.05 \\
(13.62)\end{array}$ & $\begin{array}{l}1.06 \\
(7.17)\end{array}$ & $\begin{array}{l}0.00 \\
(4.05)\end{array}$ & 100.00 \\
\hline T4 & Chlorantraniliprole $0.4 \%$ GR & 40 & 4 & $\begin{array}{l}12.96 \\
(21.42)\end{array}$ & $\begin{array}{l}11.25 \\
(19.95)\end{array}$ & $\begin{array}{l}9.58 \\
(18.42)\end{array}$ & $\begin{array}{l}7.58 \\
(16.44)\end{array}$ & $\begin{array}{l}5.14 \\
(13.68 \\
)\end{array}$ & $\begin{array}{l}3.16 \\
(10.98)\end{array}$ & 78.53 \\
\hline T5 & $\begin{array}{l}\text { Cartap Hydrochloride } 4 \% \\
\text { GR }\end{array}$ & 1000 & 10 & $\begin{array}{l}13.45 \\
(21.93)\end{array}$ & $\begin{array}{l}11.86 \\
(20.58)\end{array}$ & $\begin{array}{l}9.72 \\
(18.64)\end{array}$ & $\begin{array}{l}7.56 \\
(16.49)\end{array}$ & $\begin{array}{l}5.06 \\
(13.63 \\
)\end{array}$ & $\begin{array}{l}3.24 \\
(11.15)\end{array}$ & 77.99 \\
\hline T6 & Untreated control & & & $\begin{array}{l}13.54 \\
(22.00)\end{array}$ & $\begin{array}{c}14.12 \\
(22.47)\end{array}$ & $\begin{array}{l}14.54 \\
(22.81)\end{array}$ & $\begin{array}{l}14.59 \\
(22.85)\end{array}$ & $\begin{array}{l}14.65 \\
(22.90 \\
)\end{array}$ & $\begin{array}{l}14.72 \\
(22.95)\end{array}$ & - \\
\hline & Sem & & - & 1.09 & 0.96 & 0.82 & 0.74 & 0.54 & 0.37 & - \\
\hline & $\mathrm{CD}(\mathrm{p}=0.05)$ & & - & NS & 2.95 & 2.83 & 2.28 & 1.68 & 1.13 & - \\
\hline & $\mathrm{CV} \%$ & & - & NS & 8.50 & 8.19 & 7.97 & 7.53 & 6.87 & - \\
\hline
\end{tabular}

*Mean of three replications

Values in parenthesis are Angular transformed. 
Table.4 Bioefficacy of Spinotetram $0.8 \%$ GR on Stem borer (Second application)

\begin{tabular}{|c|c|c|c|c|c|c|c|c|c|}
\hline \multirow{2}{*}{$\begin{array}{l}\text { Sr. } \\
\text { No. }\end{array}$} & \multirow[t]{2}{*}{ Treatment } & \multirow{2}{*}{$\begin{array}{l}\text { Formulation } \\
\text { (kg or } \\
\text { ml/ha) }\end{array}$} & \multicolumn{6}{|c|}{ Percentage of White ear (\%) } & \multirow{2}{*}{$\begin{array}{l}\text { \% reduction at } \\
15 \text { days after } \\
\text { application }\end{array}$} \\
\hline & & & $\mathbf{P T}^{\wedge}$ & 3 & 7 & 10 & 14 & 21 & \\
\hline $\mathbf{T 1}$ & Spinotetram $0.8 \%$ GR & 3 & $\begin{array}{l}9.25 \\
(18.13)\end{array}$ & $\begin{array}{l}6.25 \\
(15.00)\end{array}$ & $\begin{array}{l}4.52 \\
(12.90)\end{array}$ & $\begin{array}{l}2.26 \\
(9.53)\end{array}$ & $\begin{array}{l}0.95 \\
(6.90)\end{array}$ & $\begin{array}{l}0.00 \\
(4.05)\end{array}$ & 100.00 \\
\hline $\mathbf{T} 2$ & Spinotetram $0.8 \%$ GR & 3.25 & $\begin{array}{l}9.42 \\
(18.27)\end{array}$ & $\begin{array}{l}6.35 \\
(15.10)\end{array}$ & $\begin{array}{l}4.28 \\
(12.57)\end{array}$ & $\begin{array}{l}1.95 \\
(8.97)\end{array}$ & $\begin{array}{l}0.54 \\
(5.84)\end{array}$ & $\begin{array}{l}0.00 \\
(4.05)\end{array}$ & 100.00 \\
\hline $\mathbf{T 3}$ & Spinotetram $0.8 \%$ GR & 3.5 & $\begin{array}{l}8.52 \\
(17.48)\end{array}$ & $\begin{array}{l}5.98 \\
(14.75)\end{array}$ & $\begin{array}{l}4.21 \\
(12.53)\end{array}$ & $\begin{array}{l}1.65 \\
(8.43)\end{array}$ & $\begin{array}{l}0.42 \\
(5.50)\end{array}$ & $\begin{array}{l}0.00 \\
(4.05)\end{array}$ & 100.00 \\
\hline T4 & Chlorantraniliprole $0.4 \%$ GR & 4 & $\begin{array}{l}10.05 \\
(18.86)\end{array}$ & $\begin{array}{l}7.98 \\
(16.85)\end{array}$ & $\begin{array}{l}6.58 \\
(15.36)\end{array}$ & $\begin{array}{l}4.52 \\
(12.89)\end{array}$ & $\begin{array}{l}2.98 \\
(10.71)\end{array}$ & $\begin{array}{l}1.36 \\
(7.81)\end{array}$ & 90.00 \\
\hline T5 & Cartap Hydrochloride 4\% GR & 10 & $\begin{array}{l}9.98 \\
(18.88)\end{array}$ & $\begin{array}{l}8.20 \\
(17.15)\end{array}$ & $\begin{array}{l}6.84 \\
(15.71)\end{array}$ & $\begin{array}{l}3.51 \\
(11.55)\end{array}$ & $\begin{array}{l}2.85 \\
(10.54)\end{array}$ & $\begin{array}{l}1.15 \\
(7.38)\end{array}$ & 91.54 \\
\hline T6 & Untreated control & 3 & $\begin{array}{l}13.05 \\
(21.59)\end{array}$ & $\begin{array}{l}13.25 \\
(21.76)\end{array}$ & $\begin{array}{l}13.36 \\
(21.85)\end{array}$ & $\begin{array}{l}13.42 \\
(21.90)\end{array}$ & $\begin{array}{l}13.60 \\
(22.05)\end{array}$ & $\begin{array}{l}13.60 \\
(22.05)\end{array}$ & - \\
\hline & Sem & - & 0.92 & 0.78 & 0.67 & 0.51 & 0.39 & 0.26 & - \\
\hline & $\mathrm{CD}(\mathrm{p}=\mathbf{0 . 0 5})$ & - & - & 2.39 & 2.07 & 1.58 & 1.19 & 0.81 & - \\
\hline & $\mathrm{CV} \%$ & - & - & 8.07 & 7.74 & 7.41 & 6.70 & 5.72 & - \\
\hline
\end{tabular}

*Mean of three replications

Values in parenthesis are Angular transformed. 
Table.5 Yield of Rice in various treatments of Spinotetram $0.8 \%$ GR for the management of various insect pests of Rice

\begin{tabular}{|c|c|c|c|}
\hline Sr. No. & Treatment & $\begin{array}{c}\text { Formulation(gm or } \\
\mathbf{m l / h a})\end{array}$ & $\begin{array}{c}\text { Grain Rice Yield in } \\
\mathbf{q} / \mathbf{h a}^{*}\end{array}$ \\
\hline $\mathbf{1}$ & Spinotetram 0.8\% GR & 3 & 34.28 \\
\hline $\mathbf{2}$ & Spinotetram 0.8\% GR & 3.25 & 34.89 \\
\hline $\mathbf{3}$ & Spinotetram 0.8\% GR & 3.5 & 35.24 \\
\hline $\mathbf{4}$ & Chlorantraniliprole 0.4\% GR & 4 & 25.14 \\
\hline $\mathbf{5}$ & Cartap Hydrochloride 4\% GR & 10 & 25.18 \\
\hline $\mathbf{6}$ & Untreated control & 3 & 23.85 \\
\hline & Sem & & 3.19 \\
\hline & CD (p=0.05) & & 9.82 \\
\hline & CV \% & & 17.96 \\
\hline
\end{tabular}

Table.6 Studies on the effect Spinotetram $0.8 \%$ GR on natural enemies of Rice insect pests.

\begin{tabular}{|c|c|c|c|c|c|c|c|c|}
\hline \multirow{3}{*}{$\begin{array}{l}\text { Sr. } \\
\text { No. }\end{array}$} & \multirow[t]{3}{*}{ Treatments } & \multirow{3}{*}{$\begin{array}{l}\text { Dosage } \\
\text { a.i/ha }\end{array}$} & \multicolumn{6}{|c|}{ No. of natural enemies/plant* } \\
\hline & & & \multicolumn{2}{|c|}{ Before application } & \multicolumn{2}{|c|}{$\begin{array}{c}\text { After first } \\
\text { application }\end{array}$} & \multicolumn{2}{|c|}{$\begin{array}{l}\text { After second } \\
\text { application }\end{array}$} \\
\hline & & & Spiders & Dragonflies & Spiders & Dragonflies & Spiders & Dragonflies \\
\hline 1 & $\begin{array}{c}\text { Spinotetram } 0.8 \% \\
\text { GR }\end{array}$ & 60 & $\begin{array}{c}0.62 \\
(1.06)\end{array}$ & $\begin{array}{c}0.68 \\
(1.08)\end{array}$ & $\begin{array}{c}0.64 \\
(1.07)\end{array}$ & $\begin{array}{c}0.57 \\
(1.09)\end{array}$ & $\begin{array}{c}0.68 \\
(1.08)\end{array}$ & $\begin{array}{c}0.73 \\
(1.11)\end{array}$ \\
\hline 2 & $\begin{array}{c}\text { Spinotetram } 0.8 \% \\
\text { GR }\end{array}$ & 65 & $\begin{array}{l}0.41 \\
(0.95)\end{array}$ & $\begin{array}{c}0.57 \\
(1.03)\end{array}$ & $\begin{array}{c}0.43 \\
(0.96)\end{array}$ & $\begin{array}{c}0.54 \\
(1.11)\end{array}$ & $\begin{array}{l}0.48 \\
(0.99)\end{array}$ & $\begin{array}{c}0.74 \\
(1.11)\end{array}$ \\
\hline 3 & $\begin{array}{c}\text { Spinotetram } 0.8 \% \\
\text { GR }\end{array}$ & 70 & $\begin{array}{c}0.61 \\
(1.05)\end{array}$ & $\begin{array}{c}0.70 \\
(1.10)\end{array}$ & $\begin{array}{c}0.64 \\
(1.07)\end{array}$ & $\begin{array}{c}0.57 \\
(1.12)\end{array}$ & $\begin{array}{c}0.68 \\
(1.09)\end{array}$ & $\begin{array}{c}0.78 \\
(1.13)\end{array}$ \\
\hline 4 & $\begin{array}{c}\text { Chlorantraniliprole } \\
0.4 \% \text { GR }\end{array}$ & 40 & $\begin{array}{c}0.80 \\
(1.14)\end{array}$ & $\begin{array}{c}0.55 \\
(1.02)\end{array}$ & $\begin{array}{c}0.78 \\
(1.13)\end{array}$ & $\begin{array}{c}0.60 \\
(1.01)\end{array}$ & $\begin{array}{c}0.54 \\
(1.02)\end{array}$ & $\begin{array}{c}0.51 \\
(1.00)\end{array}$ \\
\hline 5 & $\begin{array}{c}\text { Cartap } \\
\text { Hydrochloride } 4 \% \\
\text { GR }\end{array}$ & 1000 & $\begin{array}{c}0.52 \\
(1.01)\end{array}$ & $\begin{array}{c}0.71 \\
(1.10)\end{array}$ & $\begin{array}{c}0.48 \\
(0.99)\end{array}$ & $\begin{array}{c}0.61 \\
(1.07)\end{array}$ & $\begin{array}{c}0.48 \\
(0.99)\end{array}$ & $\begin{array}{c}0.58 \\
(1.04)\end{array}$ \\
\hline 6 & Untreated control & - & $\begin{array}{c}0.41 \\
(0.95)\end{array}$ & $\begin{array}{c}0.71 \\
(1.10)\end{array}$ & $\begin{array}{c}0.41 \\
(0.95)\end{array}$ & $\begin{array}{c}0.56 \\
(1.14)\end{array}$ & $\begin{array}{c}0.41 \\
(1.14)\end{array}$ & $\begin{array}{c}0.54 \\
(1.13)\end{array}$ \\
\hline & Sem & & 0.03 & 0.03 & 0.03 & 0.03 & 0.03 & 0.03 \\
\hline & $\mathrm{CD}(\mathrm{p}=\mathbf{0 . 0 5})$ & & 0.09 & NS & 0.09 & 0.09 & 0.08 & 0.09 \\
\hline & $\mathrm{CV} \%$ & & 5.04 & NS & 4.95 & 4.76 & 4.50 & 4.74 \\
\hline
\end{tabular}

*Average of 10 plants per treatment 


\section{Effect of different insecticidal treatments} on the natural enemies in rice ecosystem

Natural enemies like Spiders and Dragonflies were observed in the experimental plot before the imposition of the treatments. Before the imposition of the first application the spiders ranged from 0.41 to 0.80 and Dragonflies ranged from 0.55 to 0.80 per plot.

After the first application the spiders in Spinotetram $0.8 \%$ GR treatments ranged from 0.41 to 0.78 per plot and Dragonflies ranged from 0.54 to 0.61. After the second application spiders were 0.68 in higher dose treated plot of Spinotetram 0.8\% GR @ 70g/ha and Spinotetram 0.8\% GR @ 60g/ha and 0.48 in Spinotetram 0.8\% GR @ 65g/ha. Dragonflies were $0.78,0.74$ and 0.73 in Spinotetram 0.8\% GR @ 70g/ha, Spinotetram 0.8\% GR @ 65g/ha and Spinotetram 0.8\% GR @ 60g/ha treated plots, clearly indicating that the test molecule Spinotetram $0.8 \%$ GR was safe to the natural enemies in the Rice ecosystem (Table 6).

Among the new molecules of insecticides Carbofuran 3\% CG found to be significantly superior in reducing the per cent dead hearts and white ear heads. Earlier workers like Uthamasamy and Kuruppuchamy, (1988), Dash et.al., (1996) had similar observation like present investigation of effective control of rice pests by application of granular and sprayable insecticidal formulation. Applications of new insecticides for control of rice pest were advocated by Singh, (1993) which support the present finding.

\section{References}

Dash., A. N., Sontakke, B. K., Mukherjee, S. K., Mishra, P. R. and Rath, L. K. 1996. Efficacy of certain insecticides against major pests of rice. Oryza. 33(4):290293.

Prakash, A., David, B. V., Bambawale, O.M. 2014. Plant protection in India: Challenges and research priorities, AZRA, India. 170-174.

Singh, Y. P. 1993. Bioefficacy and residues of phorate and quinalphos in upland paddy at medium high altitude. Indian Journal of Plant Protection. 21 : 39-43.

Singh, S., Singh, B. K. 2015. survey and surveillance of major insect pest in Patna district of Bihar tate. Pro. Zoology Society India. 14(2): 69-71. 16.

Singh, S., Singh, B.K. 2017. Survey and fortnightly observation to find out major insect pests of rice crop (Oryza sativa) in Patna district of Bihar. Journal of Entomology and Zoology Studies. 5(1):766-769.

Sulagitti, A., Raghuraman, M., Reddy, M. S. S., Sathua, S. K. 2018. Impact of abiotic factors on population fluctuation of major insect pests of rice under various Condition. Exprimental Zoology, India. 21(2):709-712.

Uthamasamy, S., and Kuruppuchamy, P.1988. A note on the efficacy of new insecticides against rice pests. Indian Journal of Plant Protection. 16:265-267.

\section{How to cite this article:}

Snigdha Samanta, Mritunjoy Barman, R. Nihal and Arunava Samanta. 2020. Bio-efficacy Trials of Spinotetram 0.8\% GR against Yellow Stem Borer, Scirpophaga incertulas and Leaf Folder, Cnaphalocrocis medinalis Infesting Rice. Int.J.Curr.Microbiol.App.Sci. 9(02): 27112719. doi: https://doi.org/10.20546/ijcmas.2020.902.308 